\title{
NeuroImage
}

\section{Familiarity enhances invariance of face representations in human ventral visual cortex: fMRI evidence}

\author{
E. Eger, ${ }^{a, b, *}$ S.R. Schweinberger, ${ }^{c}$ R.J. Dolan, ${ }^{b}$ and R.N. Henson ${ }^{d}$ \\ ${ }^{a}$ Institute of Cognitive Neuroscience, University College London, UK \\ ${ }^{\mathrm{b}}$ Wellcome Department of Imaging Neuroscience, University College London, UK \\ ${ }^{\mathrm{c}}$ Department of Psychology, University of Glasgow, UK \\ ${ }^{\mathrm{d}}$ MRC Cognition and Brain Sciences Unit, Cambridge, UK
}

Received 3 November 2004; revised 9 February 2005; accepted 10 March 2005

Available online 20 April 2005

\begin{abstract}
Face recognition across different viewing conditions is strongly improved by familiarity. In the present study, we tested the hypothesis that the neural basis of this effect is a less view-dependent representation of familiar faces in ventral visual cortex by assessing priming-related fMRI repetition effects. 15 healthy volunteers made male/female judgements on familiar (famous) and unfamiliar (novel) faces preceded by the same image, a different image of the same face, or another (unprimed) face. Reaction times revealed priming by same and different images independent of familiarity and more pronounced for same than different images. In the imaging data, a main effect of prime condition was found in bilateral fusiform and orbitofrontal regions. A right anterior fusiform region expressed stronger response decreases to repetition of familiar than unfamiliar faces. Bilateral mid-fusiform areas showed stronger response decreases to repetition of same than different images. A regions-of-interest analysis focussing specifically on face responsive regions suggested differences in the degree of image dependency across fusiform cortex. Collapsing across familiarity, there was greater image dependency of repetition effects in right than left anterior fusiform, replicating previous imaging findings obtained with common objects. For familiar faces alone, there was greater generalisation of repetition effects over different images in anterior than middle fusiform. This suggests a role of anterior fusiform cortex in coding image-independent representations of familiar faces.
\end{abstract}

(C) 2005 Elsevier Inc. All rights reserved.

Keywords: Face recognition; fMRI; Invariance; Priming; Visual representation

\section{Introduction}

We are remarkably good at recognising faces across relatively low-level changes in the visual input, such as changes in lighting conditions. We also have considerable abilities to recognise faces

\footnotetext{
* Corresponding author. Institute of Cognitive Neuroscience, 17 Queen Square, London WC1N 3AR, UK. Fax: +44 2078131420.

E-mail address: e.eger@fil.ion.ucl.ac.uk (E. Eger).

Available online on ScienceDirect (www.sciencedirect.com).
}

across more complex changes, such as changes in facial expression, hairstyle, or viewing angle, when we are familiar with those faces (e.g., the faces of family or celebrities). However, we are less able to recognise faces across equivalent transformations when we are unfamiliar with them (see Hancock et al., 2000, for a review). For example, we are surprisingly inaccurate at identifying a previously seen but unknown "target" face among multiple previously unseen "distractor" faces, in conditions resembling police "line-ups" (Bruce et al., 1999; Wells, 1993). This suggests that we need to possess an "abstract" representation of a person's face, presumably following gradual learning over multiple exposures to that face, before we can identify it across different viewing conditions.

One approach towards investigating our ability to recognise faces across viewing conditions utilises the phenomenon of priming. Priming reflects a change in the behavioural response to a stimulus owing to prior exposure to the same (or a related) stimulus. The degree to which such priming effects are found despite changes in various aspects of the stimulus is used to infer how that stimulus is mentally represented. For example, if a priming effect for faces is obtained in spite of changes in lighting conditions, it might be inferred that face representations are independent of cues such as lighting that are used to extract surface properties. Conversely, if a priming effect disappears when the viewing angle is changed, it might be inferred that faces are encoded by multiple view-dependent representations. A similar logic has been used with electrophysiological (e.g., EEG) and haemodynamic (e.g., fMRI) measures of repetition effects in order to establish the temporal and spatial correlates of neural representation in the cortex (e.g., Grill-Spector et al., 1999; Henson, 2003; Naccache and Dehaene, 2001).

Behavioural effects of priming for pictures of both faces and objects often exhibit a degree of perceptual specificity, in that these effects are reduced when the perceptual format of the stimuli is changed between first and second presentation (Ellis et al., 1987; Srinivas, 1993), suggesting a degree of view-dependence of the representations mediating priming. Nonetheless, a certain degree of priming across perceptual changes usually remains, and some have suggested that abstract and view-specific representations may be 
processed in neuroanatomically separate systems, with the right hemisphere supporting view-specific priming and the left hemisphere supporting more abstract priming (Marsolek, 1995, 1999; Marsolek et al., 1992).

EEG studies measuring event-related potentials (ERPs) have found a negative deflection peaking at about $250 \mathrm{~ms}$ post-stimulus, maximal over temporal-occipital sites (using an average reference), for famous faces preceded by the same image relative to famous faces preceded by a different face (Schweinberger et al., 1995). Schweinberger et al. (2002) still found this effect, termed the "N250r", when a famous face was preceded by a different photograph of that face, though the size of the effect was diminished (relative to repetition of the same photograph). This might suggest that the neural processes giving rise to the N250r operate over both view-dependent and more abstract representations for familiar faces.

Studies using fMRI have generally found a decreased haemodynamic response associated with repetition of stimuli, so-called "repetition suppression" or "MR adaptation" (Henson, 2003). For example, Grill-Spector et al. (1999) compared mean fMRI signal during blocks of different faces with that during blocks in which the image of the same face varied in either position, size, illumination, or rotation in depth. The anterior, but not posterior, portion of the lateral occipital complex (occipito-temporal cortex that is more responsive to visual objects than scrambled objects or textures, Malach et al., $1995)$ showed adaptation across changes in position or size. No reliable adaptation was found, however, for faces presented with varying illumination or rotation, suggesting that representations of faces in this region are not viewpoint-invariant. Using immediate repetition of faces in a randomised, event-related fMRI design, Eger et al. (2004) found adaptation to faces in the mid-fusiform gyrus across changes in the spatial filtering (high-pass or low-pass) of the image, suggesting that face representations at this stage of the visualprocessing pathway also generalise across fine vs. coarse spatial frequency content. The question remains, however, whether such generalisation across viewing conditions is an automatic property of the visual system, implying that it would be found for novel stimuli, or whether it is only established after familiarisation with faces. Multiple presentations of the originally unfamiliar faces within and prior to the experiments of Eger et al. (2004) and Grill-Spector et al. (1999) might have led to the development of more abstract representations as the stimuli became familiarised.

Henson et al. (2000, 2002, 2003) directly compared fMRI repetition effects for familiar (famous) and unfamiliar (novel) faces. They found that a right mid-fusiform region exhibited repetition suppression for repeated photographs of familiar faces, but not for repeated photographs of unfamiliar faces. This suggests that representations in mid-fusiform regions are specific to the identity of known faces and do not simply represent the physical characteristics of a given image. However, this could not directly be tested as only one image of each face was used. These experiments further differed from those of Grill-Spector et al. (1999) and Eger et al. (2004) in using much longer intervals between first and second presentations of a face (with multiple intervening faces). This difference in "repetition lag" may be associated with differences in the mechanisms underlying the haemodynamic repetition effects (Henson, 2003; hence the different uses above of "adaptation" versus "repetition suppression").

Using pictures of everyday (familiar) objects, rather than faces, in a long-lag repetition paradigm, Vuilleumier et al. (2002) confirmed size independence of object representations in ventral visual cortex. In a more anterior left fusiform region, they found (unlike Grill-
Spector et al., 1999) some degree of generalisation over different views of the objects, whereas view-specificity was more pronounced on the right (see Koutstaal et al., 2001; Simons et al., 2003 for similar evidence of lateralisation using different exemplars with the same name; though see James et al. (2002) for different findings). Whether such findings generalise to faces is unclear, given arguments that the neural representation of faces differs from that of other classes of objects (Biederman and Kalocsai, 1997, but also see Tarr and Cheng, 2003 for an alternative view). Furthermore, given evidence from prosopagnosia (Landis et al., 1986) and split visual field experiments (see Rhodes, 1985) for a righthemisphere dominance in processing faces, one might in fact expect the opposite lateralisation for faces relative to objects, namely greater generalisation across face views in right than left fusiform.

The aim of the present study was to assess the influence of familiarity on the degree of view-dependence of face priming as measured by haemodynamic repetition effects. We used an immediate priming paradigm, in which participants made sex decisions to familiar (famous) and unfamiliar (novel) faces (each repeated only once to avoid confounding effects of familiarisation during the course of the experiment). The stimuli were a superset of those used by Schweinberger et al. (2002). There was no explicit control of the visual changes (such as viewing angle or illumination) across different photographs of the same person; rather, the different photographs reflected various changes more typical of everyday exposures to a person's face. The faces were presented in a continuous sequence, in which a face was either preceded by the same photograph (same image trials), a different photograph of the same face (different image trials), or a different face of the same sex (unprimed trials). The order of trials was randomised together with additional single face (filler) trials that served to break-up the pattern of pairs of faces with the same sex. The experiment thus conformed to a $2 \times 3$ factorial design with two levels of familiarity (familiar and unfamiliar faces) and three levels of priming (same image, different image, and unprimed; Fig. 1).

Given the evidence reviewed above, we had three main predictions. Firstly, based on behavioural evidence for better recognition across views for familiar than unfamiliar faces (Hancock et al., 2000), we expected to find less image dependency of behavioural priming effects for familiar than unfamiliar faces, with a similar pattern in the priming-related haemodynamic response reductions in fusiform cortex (Henson et al., 2003). Secondly, based on Grill-Spector et al. (1999) and Vuilleumier et al. (2002), we expected less image dependency of repetition effects in anterior than posterior fusiform regions. Finally, if faces are processed similarly to objects, we expected less image dependency of repetition effects in left than right fusiform, based on Marsolek's (1999) and Vuilleumier et al. (2002) experiments using objects (alternatively, if faces are processed differently from objects, we might expect the opposite lateralisation instead, based on the right-lateralised processing of faces suggested by Landis et al., 1986; Rhodes, 1985).

\section{Materials and methods}

\section{Participants}

Fifteen right-handed healthy young volunteers (4 men and 11 women, mean age $21.8 \pm 2.8$ years) with normal or corrected- 
A
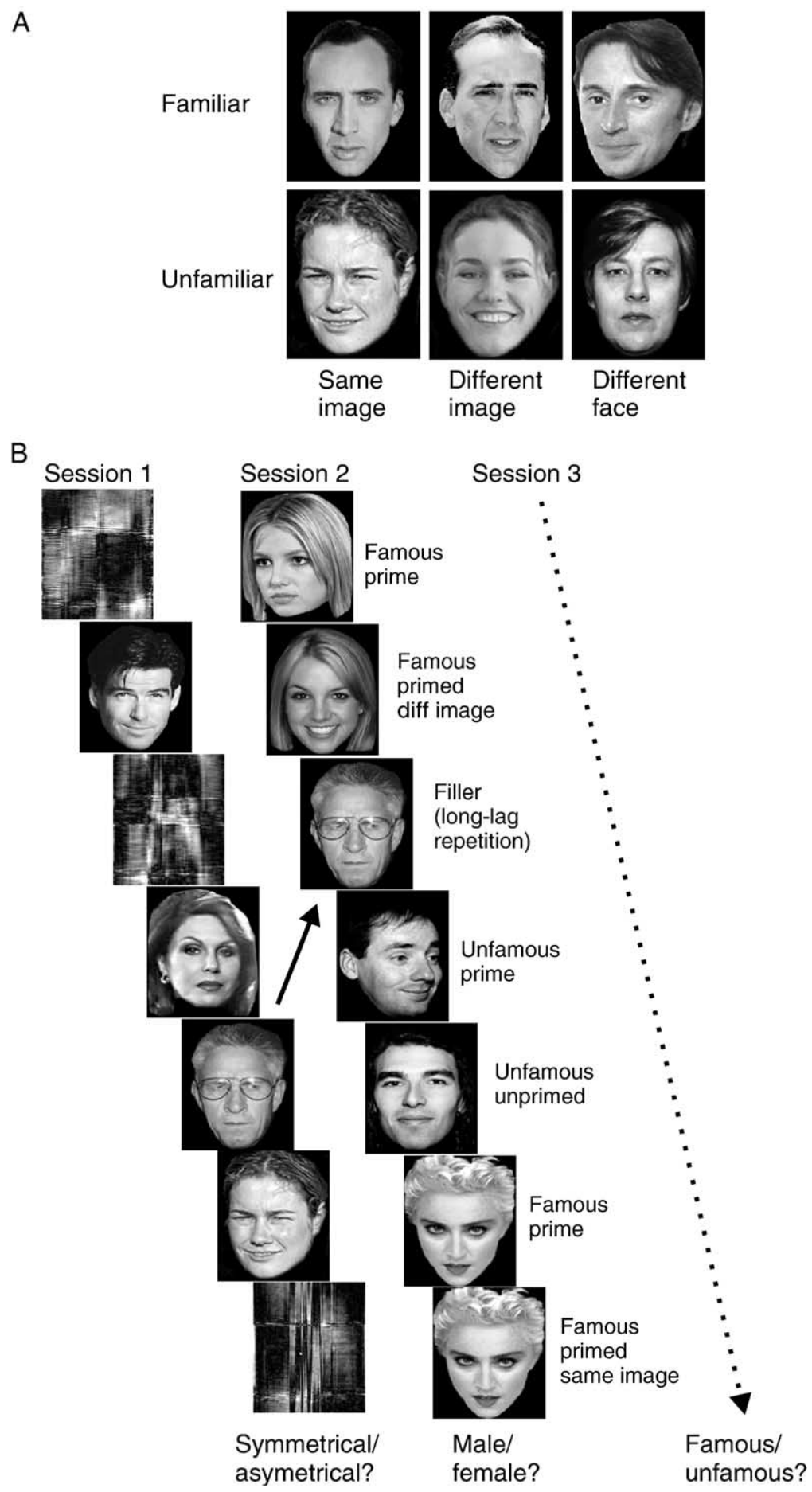

Fig. 1. Stimuli and experimental design. (A) The $2 \times 3$ factorial design included famous and unfamous faces immediately preceded by either the same image, a different image of the same face, or a different (unprimed condition) face of the same sex. (B) In the first session, participants viewed pictures of faces or “scrambled" faces while performing a symmetry judgement on these stimuli. In the second (main) session, the participants' task was to make male/female sex judgements on faces corresponding to the experimental conditions mentioned above. These relevant trials were interspersed by single filler faces (repetitions from session 1) that served to break up the pattern of same-sex trial pairs. The purpose of the third session, in which all stimuli from the second session were repeated, was to obtain familiarity judgements for all the faces.

to-normal vision and no history of neurological or ophthalmological disease participated in the study after giving written informed consent. The study was of the type approved by university ethics committees (references: UCL/UCLH 99/0048, NH/ION 00/N031).

\section{Basic design}

The experiment consisted of three sessions. In a first session, participants made symmetry judgements to faces and scrambled faces, which functioned as a localiser to identify regions of interest, 
and as a mask to restrict subsequent contrasts to face-responsive regions (reducing the likelihood of type I errors). The main experimental hypotheses were tested in the second session, in which participants made sex judgements to faces, some of which were repeated immediately in the same or a different image (see Introduction). The final session involved re-presenting all the faces from the second session (though in a different order), but this time participants made a familiarity judgement to each face. The purpose of this last session was to define the familiar/unfamiliar conditions according to the participant's own experience. In other words, famous faces were only classified as "familiar" for a given subject if that subject recognised (both views of) the corresponding person. Famous faces not recognised, and unfamous faces erroneously "recognised", were ignored in the analysis.

\section{Materials}

Stimuli were greyscale photos of faces. There were two images each of 120 famous and 120 non-famous people, half male and half female, derived from a superset of those used by Schweinberger et al. (2002). The photos were matched for image size, cropped to show face and hair only. There was no explicit control of the differences across the two images: The two photographs could be taken from different perspectives (though the majority were between frontal and three-quarters views), involve different facial expressions and/or differences in lighting conditions, or hairstyles. There was little difference across the two photographs in the age of the person. Unfamiliar faces were matched to famous counterparts with respect to gender and approximate age. The celebrities used in this study were selected on the basis of high ratings for ease of face recognition in a preliminary study, scoring an average of 1.95 out of 2 (range: $1.73-2.00$; means across 11 raters, scores are on a 3point scale labelled 0 -would not recognise; 1 -may perhaps recognise; 2 -would recognise). An attempt was also made to homogenise the stimuli with respect to average luminance and contrast. The scrambled faces in the first session were created by a Fourier transform of the original images, random permutation of the phase information, and inverse transformation. The scrambled faces were therefore matched for spatial frequency power density.

The faces were randomly split into 5 sets of 24 (one set for the Primed Same condition, one for Primed Different, two for Unprimed and one for the fillers). The assignment of these face sets to the conditions was rotated across participants to control for stimulus differences.

\section{Procedure}

The stimuli were displayed for $500 \mathrm{~ms}$ with an SOA of $3120 \mathrm{~ms}$ against a black background, projected onto a screen approximately $65 \mathrm{~cm}$ behind the participant, which they viewed via a pair of mirrors placed above the eyes. The black square subtended a horizontal visual angle of approximately $4^{\circ}$ and a vertical visual angle of approximately $5^{\circ}$.

In the first session, the participants' task was to decide whether each stimulus (face or scrambled) was more symmetrical or more asymmetrical (a subjective decision, as used by Henson et al., 2003). In the second session, their task was to decide, as quickly as possible, whether each face was male or female. Note that unprimed trial pairs consisted of faces with the same sex (so priming was not confounded by response differences) and that the single filler trials prevented subjects from being able to predict the sex of the next face on the basis of the sex of the current face. Some filler trials were also faces that were repeated from the first (localiser) session, preventing subjects from expecting that all repetitions would be immediate.

In the third session, the subjects' task was to make a familiarity judgement. They were told that, in order to respond "familiar", they did not have to be able to name the faces but should be sure that they had seen the face somewhere before the experiment began (so as to minimise confusion between pre-experimental familiarity and experimentally induced familiarity owing to repetition). For all tasks, participants responded by pressing a key with the index finger of either their left or right hand, with both speed and accuracy emphasised. The assignment of hand to response alternated across participants. Participants were told that some faces were repeated in the second and third sessions but that this did not affect their task. Each participant received a short practice session prior to scanning. The whole experiment lasted approximately $40 \mathrm{~min}$.

\section{fMRI acquisition}

A 3 T Allegra system (Siemens, Erlangen, Germany) was used to acquire $32 \mathrm{~T} 2 *$-weighted transverse echoplanar images (EPI) $(64 \times 643 \times 3 \mathrm{~mm}$ pixels, $\mathrm{TE}=30 \mathrm{~ms})$ per volume with blood oxygenation level dependent (BOLD) contrast. EPIs comprised 2 mm-thick axial slices taken every $3.5 \mathrm{~mm}$, with a pitch of $30^{\circ}$ up at the front (to minimise eye-ghosting). The slices were acquired in an even-odd interleaved descending direction. A total of three sessions of 155,515 , and 515 volumes respectively were collected continuously with a repetition time (TR) of $2080 \mathrm{~ms}$. The first 5 volumes per session were discarded to allow for equilibration effects. The ratio of SOA to TR ensured that the impulse response was sampled every $1040 \mathrm{~ms}$ (over trials).

\section{Behavioural analysis}

Trials of interest in the second session were restricted to correct sex decisions and correct "familiar/unfamiliar" classification according to the familiarity judgements in the third session (see Materials and methods). Data were analysed with repeated measures analysis of variance (ANOVA) using a GreenhouseGeisser correction. Effects in the presence of higher order interactions are not reported. Further planned comparisons were tested for "image-dependent" priming (Same versus Unprimed conditions) and "image-independent" priming (Different versus Unprimed conditions), as well as the difference in both (Same versus Different)

\section{fMRI analysis}

Data were analyzed using statistical parametric mapping software (SPM2, www.fil.ion.ucl.ac.uk/spm2.html). Preprocessing of the image volumes included standard realignment and unwarping to correct for additional movement-by-susceptibility-interactions, slice-time correction with reference to the middle slice acquired in time, normalisation to the EPI-template included in SPM2 (re-sampled voxel size $3 \mathrm{~mm}$ ), and spatial smoothing by a 10 mm FWHM Gaussian kernel.

Responses to all experimental conditions were modelled by delta functions marking stimulus onset convolved with the canonical haemodynamic response function (HRF) as implemented in 
SPM2. Faces given incorrect sex decisions or incorrect familiarity judgements (from the third session) were modelled as separate regressors. To account for residual movement artifacts after realignment, the model also included an additional covariate of no interest marking scans that include signal outliers (defined as slices whose mean or variance of voxel values was more than 5 standard deviations of the average mean/variance for that slice across all scans after realignment).

Voxel-wise parameter estimates for these regressors were obtained by maximum-likelihood estimation, while using a temporal high-pass filter (cut-off $128 \mathrm{~s}$ ) to remove low-frequency drifts, and modelling temporal autocorrelation across scans with an $\mathrm{AR}(1)$ process. The comparisons of interest were performed as random-effects group analyses on contrast images for each subject.

Main effects and interactions in the factorial design were tested by F-contrasts and were masked for the T-contrast of faces $>$ scrambled faces in the localiser scan (at $P<0.001$, uncorrected). The SPMs for the main effects were exclusively masked with the SPM for the interaction at $P<0.05$ uncorrected, given that main effects are uninterpretable in the presence of interactions. Further planned comparisons of image-specific and image-independent priming effects employed T-contrasts, masked both by the main effect of priming and the face localiser (at $P<0.001$, uncorrected). "Image-dependent" effects were defined by the contrast unprimed $>$ same image, masked exclusively by unprimed $>$ different image, whereas "image-independent" effects were defined by the contrast different image $>$ unprimed, masked inclusively by same image $>$ unprimed (all $P<0.001$, uncorrected). The stereotactical coordinates reported are based on the Montreal Neurological Institute (MNI) brain, which bear a close, but not exact, correspondence to the atlas of Talairach and Tournoux.

For the regions of interest (ROI) analysis, parameter estimates for the different conditions for each subject were averaged across the voxels included in spherical volumes of $5 \mathrm{~mm}$ radius centered on mid- and anterior fusiform group maxima from the localiser scan (faces $>$ scrambled faces). Data were analysed with repeated measures analysis of variance (ANOVA) using a GreenhouseGeisser correction. Effects in the presence of higher order interactions are not reported; higher order interactions were broken down by further ANOVAs. For effects that conformed to one of our directional predictions (see Introduction), we accepted significance based on one-tailed $t$ tests.

\section{Results}

\section{Behavioural results}

Less than $10 \%$ of faces were given incorrect sex decisions; and less than $15 \%$ were given incorrect familiarity judgements. Reaction times for the six conditions of interest are shown in Table

Table 1

Behavioural results

\begin{tabular}{llll}
\hline & Same image & Different image & Unprimed \\
\hline Unfamiliar & $518(27.7)$ & $552(28.5)$ & $608(31.1)$ \\
Familiar & $495(27.3)$ & $551(26.7)$ & $584(26.8)$ \\
\hline
\end{tabular}

Mean $(n=15)$ and standard error (in smooth parentheses) of median RTs (ms) as a function of familiarity and prime condition.
1. The repeated measures ANOVA with factors of prime condition (same, different, unprimed) and familiarity (familiar, unfamiliar) revealed a main effect of prime condition $(F(1.63,22.81)=55.4$, $P<0.0001)$, without a significant interaction $(F(1.72,24.17)=$ 1.24, $P=0.30)$, and a marginal tendency for a main effect of familiarity $(F(1,14)=3.77, P=0.073)$. One-tailed $t$ tests confirmed significant effects of both image-dependent priming (Same versus Unprimed conditions; familiar faces: $t(14)=7.16, P<0.0001$, unfamiliar faces: $t(14)=5.88, P<0.0001)$ and image-independent priming (Different versus Unprimed conditions; familiar faces: $t(14)=2.79, P<0.01$, unfamiliar faces: $t(14)=5.19, P<$ $0.0001)$, as well as an additional significant facilitation due to priming with same compared to different images (Same versus Different conditions; familiar faces: $t(14)=8.81, P<0.0001$, unfamiliar faces: $t(14)=2.75, P<0.01)$.

\section{fMRI results}

Since we were particularly interested in the effect of repetition on processes related to face perception, we masked comparisons of interest by the localiser-contrast of faces $>$ scrambled faces at $P<0.001$, uncorrected. The regions significant in this comparison are summarised in Table 2. They included fusiform/lateral occipital regions usually found activated for faces in comparison with common objects (e.g., Kanwisher et al., 1997; Sergent et al., 1992) or scrambled faces (e.g., Puce et al., 1995). These ventral temporal activations are believed to reflect processing of facial identity (Hoffman and Haxby, 2000), although their degree of "face-specificity" remains controversial (e.g., Gauthier et al., 1999).

\section{Whole-brain SPM analyses}

Imaging data from the main experimental session were first assessed according to the main effects of the experimental factors of prime condition and familiarity (Table 3). In these statistical comparisons, we excluded areas that also showed an interaction of both factors by exclusively masking these main effects with the interaction-SPM at $P<0.05$, uncorrected.

A main effect of prime condition was found in left and right mid- to anterior fusiform areas, as well as in regions of orbitofrontal cortex (Fig. 2A, Table 3). All of these regions expressed decreased BOLD responses for primed relative to unprimed faces.

A main effect of familiarity was evident in the retrosplenial/ posterior cingulate cortex, left hippocampus, and orbitofrontal cortex (Fig. 2B, Table 3), as well as in the left and right temporal poles, left fusiform, and a further frontal region. All of these regions expressed BOLD signal increases for familiar relative to unfamiliar faces. An interaction between prime condition and familiarity was found in the right hippocampus, a medial part of right anterior fusiform cortex, as well as in the left anterior STS and a left basal ganglia region close to the hippocampus (Fig. 3, Table 3). In all these regions, the direction of the effect was towards more pronounced priming-related decreases for familiar than unfamiliar faces. For the right anterior fusiform region, separate tests showed a significant effect of prime condition for famous $(Z=4.8)$, but not unfamous $(Z=0.65)$ faces. As the plot in Fig. 3 indicates, this region expresses repetition decreases mainly for same images of familiar faces. 
Table 2

Results of the face-localiser in SPM2 group analysis $(n=15)$

\begin{tabular}{|c|c|c|c|c|}
\hline \multirow{2}{*}{$\frac{\text { Region }}{\text { Faces }>\text { 'scrambled face }}$} & \multicolumn{3}{|c|}{ Stereotactic coordinates } & \multirow[t]{2}{*}{$Z$} \\
\hline & & & & \\
\hline \multirow{3}{*}{$\begin{array}{l}\text { Right fusiform and } \\
\text { lateral occipital }\end{array}$} & 45 & -45 & -24 & $5.73 *$ \\
\hline & 42 & -63 & -15 & $5.05 *$ \\
\hline & 45 & -84 & -9 & $5.30 *$ \\
\hline \multirow[t]{2}{*}{ Left fusiform } & -39 & -39 & -27 & 4.27 \\
\hline & -48 & -51 & -18 & 3.54 \\
\hline \multirow[t]{3}{*}{ Right hippocampus } & 24 & -18 & -18 & $5.29 *$ \\
\hline & 30 & -3 & -42 & $5.01 *$ \\
\hline & 27 & 0 & -24 & 3.83 \\
\hline Left hippocampus & -27 & -6 & -30 & $4.84 *$ \\
\hline \multirow[t]{3}{*}{ Orbitofrontal cortex } & -3 & 51 & -12 & $5.00 *$ \\
\hline & -9 & 42 & -21 & 4.68 \\
\hline & -3 & 57 & -3 & 4.36 \\
\hline \multirow{3}{*}{$\begin{array}{l}\text { Superior medial } \\
\text { prefrontal cortex }\end{array}$} & -12 & 33 & 60 & $4.91 *$ \\
\hline & -3 & 60 & 39 & $4.80 *$ \\
\hline & 0 & 54 & 27 & 4.48 \\
\hline $\begin{array}{l}\text { Retrosplenial/posterior } \\
\text { cingulate cortex }\end{array}$ & -6 & -57 & 30 & 4.80 \\
\hline Left temporal pole & -42 & 12 & -36 & 4.11 \\
\hline \multirow[t]{2}{*}{ Right temporal pole } & 51 & 15 & -39 & 3.92 \\
\hline & 54 & 15 & -30 & 3.48 \\
\hline Left anterior STS & -66 & -24 & -21 & $5.14 *$ \\
\hline Right anterior STS & 54 & -6 & -24 & 3.80 \\
\hline \multirow[t]{2}{*}{ Left posterior STS } & -63 & -42 & 3 & 3.70 \\
\hline & -66 & -51 & 6 & 3.61 \\
\hline \multirow[t]{3}{*}{ Left inferior parietal } & -42 & -66 & 24 & 3.59 \\
\hline & -54 & -66 & 35 & 3.51 \\
\hline & -51 & -75 & 24 & 3.22 \\
\hline \multirow[t]{3}{*}{ Left lateral prefrontal } & -39 & 33 & -15 & $4.90 *$ \\
\hline & -27 & 27 & -21 & 3.81 \\
\hline & -45 & 30 & 6 & 3.48 \\
\hline \multirow[t]{2}{*}{ Right lateral prefrontal } & 57 & 30 & 9 & 4.13 \\
\hline & 36 & 36 & -18 & 4.00 \\
\hline
\end{tabular}

Clusters that exceed an extend threshold of 10 voxels at $P<0.001$, uncorrected, are reported. Results that survive correction for the whole brain at $P<0.05$ at voxel-level are indicated by an asterisk.

To further investigate whether priming effects were sensitive to changes in the image of the face, we tested pairwise planned comparisons within the main effect of priming for familiar and unfamiliar faces. Image-dependent repetition decreases (as defined by the contrast of unprimed $>$ same image conditions, masked exclusively by unprimed $>$ different image conditions at $P<$ 0.001 , uncorrected) were found for familiar faces in the bilateral fusiform gyri, as well as in a medial orbitofrontal region (Table 4, Fig. 4A). The same comparison also reached significance in bilateral mid-fusiform areas for unfamiliar faces (Table 4, Fig. 4B). The test for image-independent effects (unprimed $>$ different image conditions, masked inclusively by unprimed $>$ same image conditions at $P<0.001$, uncorrected) reached significance, for familiar faces, in a left anterior fusiform region, as well as in orbitofrontal cortex (Table 4, Fig. 4C). No voxels did survive our predefined significance threshold for unfamiliar faces in this comparison.

\section{ROI analyses and topographic differences}

Given the above patterns to suggest that familiarity and image changes might exert differential effects on priming in subregions of fusiform cortex and to test whether there was a significant lateralisation of the effects of image changes (as already suggested by Vuilleumier et al., 2002, for objects), we performed further analyses of fusiform repetition effects in regions of interest defined by independent data, namely the face-localiser contrast. We extracted the parameter estimates from the four fusiform response maxima in this contrast $(+45-45-24,+42$ $-63-15,-39-39-27,-48-51-18$, see Materials and methods for details) and subjected them to an ANOVA with the additional factors of rostrality (middle/anterior) and laterality (left/ right). Note that, though functionally defined on the basis of independent data, these ROIs are still derived from locations of group-maxima within normalised anatomical space, rather than from individually defined maxima (cf. Kanwisher et al., 1997). This reflects our working assumption of a single functionalanatomical mapping within the population as a whole (that exists at the spatial scale of the anatomical normalisation, Ashburner and Friston, 1999).

The resulting $2 \times 2 \times 2 \times 3$ ANOVA revealed a significant threeway interaction between rostrality, familiarity, and priming, $F(1.91,26.7)=3.43, P<0.05$, and a significant three-way interaction between laterality, rostrality, and priming, $F(1.75,24.5)=$ $3.80, P<0.05$. As above, in order to investigate these interactions in more detail, priming effects were examined in further $2 \times 2 \times 2$

Table 3

Results of SPM2 group analysis $(n=15)$

\begin{tabular}{|c|c|c|c|c|c|}
\hline \multirow{2}{*}{$\frac{\text { Contrast }}{\text { Main effects }}$} & \multirow[t]{2}{*}{ Region } & \multicolumn{3}{|c|}{ Stereotactic coordinates } & \multirow[t]{2}{*}{$Z$} \\
\hline & & & & & \\
\hline \multirow[t]{9}{*}{ Priming } & \multirow[t]{3}{*}{ Right fusiform gyrus } & 39 & -63 & -15 & 4.08 \\
\hline & & 39 & -51 & -18 & 3.90 \\
\hline & & 45 & -42 & -18 & 3.76 \\
\hline & \multirow[t]{3}{*}{ Left fusiform gyrus } & -45 & -48 & -18 & 3.83 \\
\hline & & -42 & -42 & -21 & 3.72 \\
\hline & & -33 & -39 & -30 & \\
\hline & \multirow[t]{3}{*}{ Orbitofrontal cortex } & -6 & 33 & -27 & 3.51 \\
\hline & & -3 & 36 & -24 & $3.4 \mathrm{C}$ \\
\hline & & -12 & 45 & -15 & \\
\hline \multirow[t]{14}{*}{ Familiarity } & \multirow[t]{2}{*}{ Left hippocampus } & -30 & -12 & -24 & \\
\hline & & -27 & -3 & -33 & 3.42 \\
\hline & \multirow[t]{3}{*}{ Orbitofrontal cortex } & -9 & 45 & -21 & 4.93 \\
\hline & & -6 & 63 & -9 & 4.69 \\
\hline & & -9 & 54 & -15 & 4.20 \\
\hline & \multirow{2}{*}{$\begin{array}{l}\text { Retrosplenial/posterior } \\
\text { cingulate cortex }\end{array}$} & 6 & -60 & 33 & \\
\hline & & 3 & -57 & 24 & \\
\hline & \multirow[t]{3}{*}{ Left temporal pole } & -36 & 18 & -33 & \\
\hline & & -33 & 21 & -27 & \\
\hline & & -30 & 24 & -24 & \\
\hline & Right temporal pole & 33 & 6 & -45 & 3.49 \\
\hline & Left fusiform gyrus & -48 & -48 & -21 & 3.42 \\
\hline & \multirow[t]{2}{*}{ Left frontal operculum } & -30 & 33 & -12 & 3.27 \\
\hline & & -36 & 30 & -12 & \\
\hline \multicolumn{6}{|l|}{ Interaction } \\
\hline \multirow{5}{*}{$\begin{array}{l}\text { Priming } \times \\
\quad \text { familiarity }\end{array}$} & Right hippocampus & 27 & -27 & -18 & \\
\hline & Left anterior STS & -57 & -6 & -24 & \\
\hline & Right fusiform gyrus & 33 & -42 & -24 & \\
\hline & Left basal ganglia & -18 & -12 & -15 & 3.1 \\
\hline & (Above hippocampus) & -24 & -9 & -18 & \\
\hline
\end{tabular}

F-contrasts of main effects and interaction in factorial design (masked inclusively with the face localiser at $P<0.001$, uncorrected). Results of the main effects are reported masked exclusively by the interaction-SPM at $P<$ 0.05 , uncorrected. 

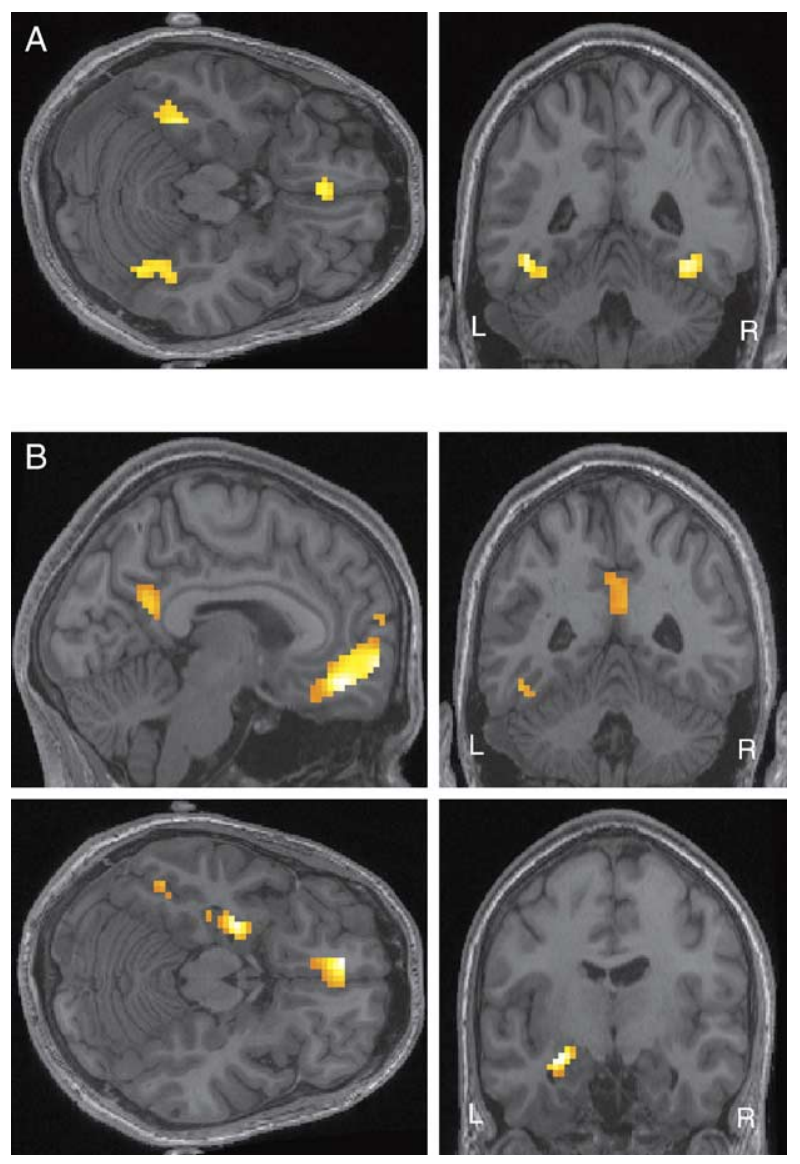

Fig. 2. Main effect of priming (A) and familiarity (B). SPMs of F-contrasts in a group analysis $(n=15)$ were overlaid on the structural scan of one participating subject. For visualisation, effects were color-coded at $P<$ 0.001 , uncorrected, and masked inclusively with the face localiser at $P<$ 0.001 , uncorrected. The main effect of priming revealed significant activations in bilateral fusiform cortex and in orbitofrontal cortex. The main effect of familiarity was significant in the left fusiform, left hippocampus, orbitofrontal cortex, and retrosplenial/posterior cingulate cortex.

ANOVAs for same image versus unprimed and different image versus unprimed, respectively.

To examine the interaction of priming with rostrality and familiarity, data were collapsed over laterality. The ANOVA for image-dependent priming effects (same image versus unprimed) showed a significant interaction between priming, familiarity, and rostrality, $F(1,14)=7.4, P<0.05$. To examine this pattern in more detail, separate ANOVAs were performed for familiar and unfamiliar faces. For familiar faces, this reflected a trend for greater image-dependent priming in anterior than middle fusiform regions, $F(1,14)=4.1, P=0.06$, though same image priming was significant in both middle, $F(1,14)=19.2, P<0.001$, and anterior, $F(1,14)=40.2, P<0.001$, fusiform (Fig. 5). For unfamiliar faces, only a significant main effect of image-dependent priming, $F(1,14)=8.6, P<0.01$, was found, but no interaction with rostrality, $F(1,14)=1.9$.

The ANOVA for image-independent effects showed an interaction between rostrality, familiarity, and different image versus unprimed conditions, $t(14)=1.93, P<0.05$, one-tailed, that was significant given our directional prediction (see Introduc- tion) for greater image-independent priming effects for familiar than unfamiliar faces in anterior fusiform regions compared with middle fusiform regions (Fig. 5). To confirm this pattern in more detail, separate ANOVAs were again performed for familiar and unfamiliar faces. For familiar faces, there was an interaction between rostrality and image-independent priming, $F(1,14)=5.13$, $P<0.05$, for which simple effects showed an image-independent priming effect in anterior, $F(1,14)=5.62, P<0.05$, but not middle, $F(1,14)<1$, fusiform regions. For unfamiliar faces, there were no significant effects of image-independent priming or rostrality, $F(1,14)<1.98$.

To examine the interaction of priming with laterality and rostrality, data were collapsed over familiarity. The ANOVA for image-dependent effects showed an interaction between laterality and same image versus unprimed, $F(1,14)=4.78, P<0.05$. This reflected greater same-image effects in the right than left fusiform, though image-dependent priming was significant in both right, $F(1,14)=23.6, P<0.001$, and left, $F(1,14)=18.1, P<0.001$, fusiform (Fig. 5).

The ANOVA for image-independent effects showed a threeway interaction between laterality, rostrality, and different image versus unprimed conditions, $t(14)=1.99, P<0.05$, one-tailed, given the predictions of Vuilleumier et al. (2002); see Introduction. Follow-up ANOVAs were performed on anterior and middle regions separately. In anterior fusiform regions, there was a significant main effect of image-independent priming, $t(14)=$ 2.09, $P<0.05$, one-tailed, given the predicted response decrease associated with priming, but no interaction with laterality. There were no significant effects of image-independent priming in the middle fusiform regions $(F(1,14)=1.7)$.

Finally, since the contrast of same-image versus unprimed reflects the "overall" priming effect (part of which might also be obtained by a different image), we further asked whether the "gain" in the amount of repetition decrease for the same versus a different image differed across regions. Thus, for the two three-way interaction results obtained, we also re-examined whether there was a difference between priming due to same image and different image.

For the interaction of priming with familiarity and rostrality, follow-up tests revealed a significant interaction between familiarity and same versus different image, $F(1,14)=7.09$, $P<0.05$. This reflected a greater difference for familiar than unfamiliar faces, which was not expected, though simple effects were significant for both familiar, $F(1,14)=15.1, P<0.005$, and unfamiliar, $F(1,14)=5.22, P<0.05$, faces. No significant interaction with rostrality was observed, $F(1,14)<1$. This indicates that the anterior-posterior difference in image-dependent effects for familiar faces found above reflects a difference in the overall priming effect and not a relative difference in the image-specific component.

For the interaction of priming with laterality and rostrality, we also found a significant interaction of same versus different image with laterality and rostrality, $F(1,14)=6.32, P<0.03$. Follow-up tests showed a significant main effect of same versus different image in the middle fusiform, $F(1,14)=23.03, P<0.001$, reflecting more pronounced repetition decreases for same than different image (see Fig. 5), but no interaction with laterality, $F(1,14)<1$. In the anterior fusiform, however, there was a significant interaction between same versus different image and laterality, $F(1,14)=12.94, P<0.005$, for which follow-up tests indicated a difference between same and different image priming in 

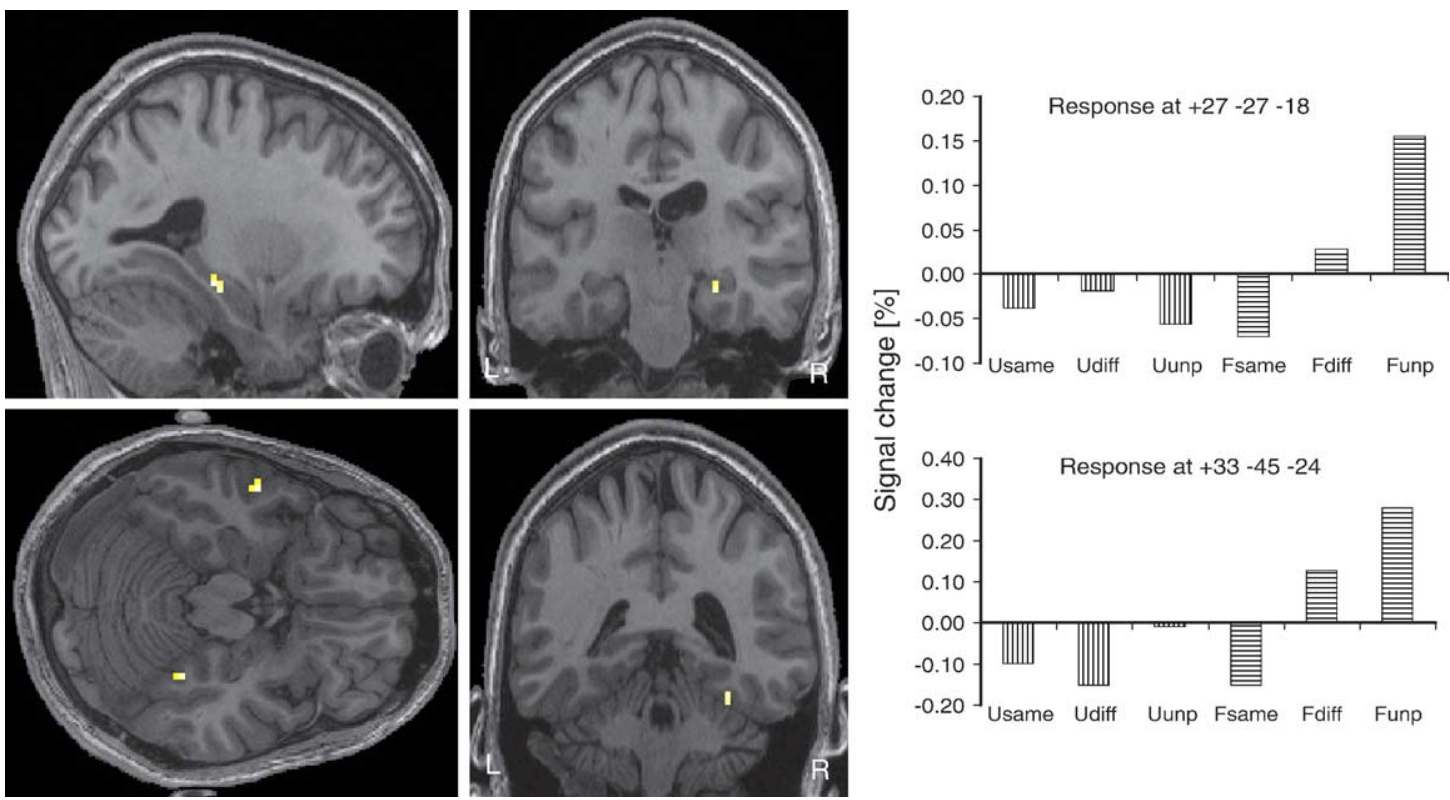

Fig. 3. Priming by familiarity interaction. The SPM of an F-contrast in a group analysis $(n=15)$ at $P<0.001$, uncorrected, masked inclusively with the face localiser at $P<0.001$, uncorrected, was overlaid on the structural scan of one participating subject. The plots show the effect size (contrast of parameter estimates) for the different experimental conditions in the hippocampal and fusiform region shown, indicating that repetition decreases are more pronounced for familiar faces (note that the "zero" on the $y$ axis, i.e., the response versus interstimulus baseline, was not estimated reliably in this rapid, short-SOA design).

the right fusiform, $F(1,14)=11.63, P<0.005$, which did not reach significance in the left fusiform, $F(1,14)=1.32, P=0.27$. Priming with the same as compared to a different image therefore yields a stronger repetition-related decrease in the middle fusiform, and in

Table 4

Results of SPM2 group analysis $(n=15)$

\begin{tabular}{|c|c|c|c|c|c|}
\hline \multirow{3}{*}{$\begin{array}{l}\text { Contrast } \\
\text { Image-specific } \\
\text { priming (familiar) }\end{array}$} & \multirow{4}{*}{$\begin{array}{l}\text { Region } \\
\begin{array}{l}\text { Right fusiform } \\
\text { gyrus }\end{array}\end{array}$} & \multicolumn{3}{|c|}{ Stereotactic coordinates } & \multirow{2}{*}{$\begin{array}{l}Z \\
4.91\end{array}$} \\
\hline & & 36 & -45 & -21 & \\
\hline & & 45 & -63 & -18 & 3.66 \\
\hline & & 42 & -72 & -15 & 3.44 \\
\hline & Left fusiform & -36 & -36 & -30 & 4.65 \\
\hline & gyrus & -45 & -48 & -21 & 3.66 \\
\hline & $\begin{array}{l}\text { Orbitofrontal } \\
\text { cortex }\end{array}$ & 0 & 33 & -27 & 4.16 \\
\hline \multirow[t]{2}{*}{$\begin{array}{l}\text { Image-independent } \\
\text { priming (familiar) }\end{array}$} & $\begin{array}{l}\text { Left fusiform } \\
\text { gyrus }\end{array}$ & -30 & -33 & -27 & 3.17 \\
\hline & $\begin{array}{l}\text { Orbitofrontal } \\
\text { cortex }\end{array}$ & 3 & -36 & -24 & 3.08 \\
\hline \multirow{2}{*}{$\begin{array}{l}\text { Image-specific } \\
\text { priming } \\
\text { (unfamiliar) }\end{array}$} & $\begin{array}{l}\text { Left fusiform } \\
\text { gyrus }\end{array}$ & -45 & -48 & -18 & 3.24 \\
\hline & $\begin{array}{l}\text { Right fusiform } \\
\text { gyrus }\end{array}$ & 42 & -54 & -15 & 3.04 \\
\hline $\begin{array}{l}\text { Image-independent } \\
\text { priming } \\
\text { (unfamiliar) }\end{array}$ & $\begin{array}{l}\text { No suprathreshold } \\
\text { voxels }\end{array}$ & & & & \\
\hline
\end{tabular}

Image-specific and image-independent priming effects for familiar and unfamiliar faces. Image-specific effects correspond to t-contrasts of same image $>$ unprimed, masked exclusively by different image $>$ unprimed at $P<0.001$, uncorrected. Image-independent effects correspond to t-contrasts of different image $>$ unprimed, masked inclusively by same image $>$ unprimed at $P<0.001$, uncorrected. All comparisons masked inclusively by the face-localiser and the main-effect of prime condition $(P<0.001$, uncorrected). Clusters that exceed a threshold of $P<0.001$, uncorrected, are reported. the right anterior fusiform, but not the left anterior fusiform (see Fig. 5).

\section{Image dissimilarity}

To address the possibility that more pronounced imageindependent effects for familiar than unfamiliar faces could be related to an unequal degree of image change across the two photographs for familiar and unfamiliar faces, we computed dissimilarity indices for all faces (see Grill-Spector et al., 1999 and Vuilleumier et al., 2002). The dissimilarity measure corresponds to the root mean-square difference in grey level between two images, scaled to have a maximum of 1 . The indexed change was on average $0.0008 \pm 0.0003$ between same and different photographs of unfamiliar faces and $0.0013 \pm 0.0003$ between same and different photographs of familiar faces. The greater mean dissimilarity for familiar than unfamiliar faces was highly significant $(t(238)=13.8, P<0.001)$, but in the opposite direction to that necessary to explain the greater generalisation of priming over image changes for familiar than unfamiliar faces (e.g., in anterior fusiform).

\section{Discussion}

The present study manipulated face familiarity and image changes between prime and probe in an orthogonal fashion to determine the extent to which priming-related repetition decreases in fMRI signal generalise across different images and whether such generalisation is related to the degree of familiarity of a given face (given behavioural evidence that we are much better at generalising over views for familiar than unfamiliar faces). A large set of stimuli was used, in which two different "views" could differ along several dimensions, such as rotational angle, expression, lighting, among others. 


\section{Image-specific}
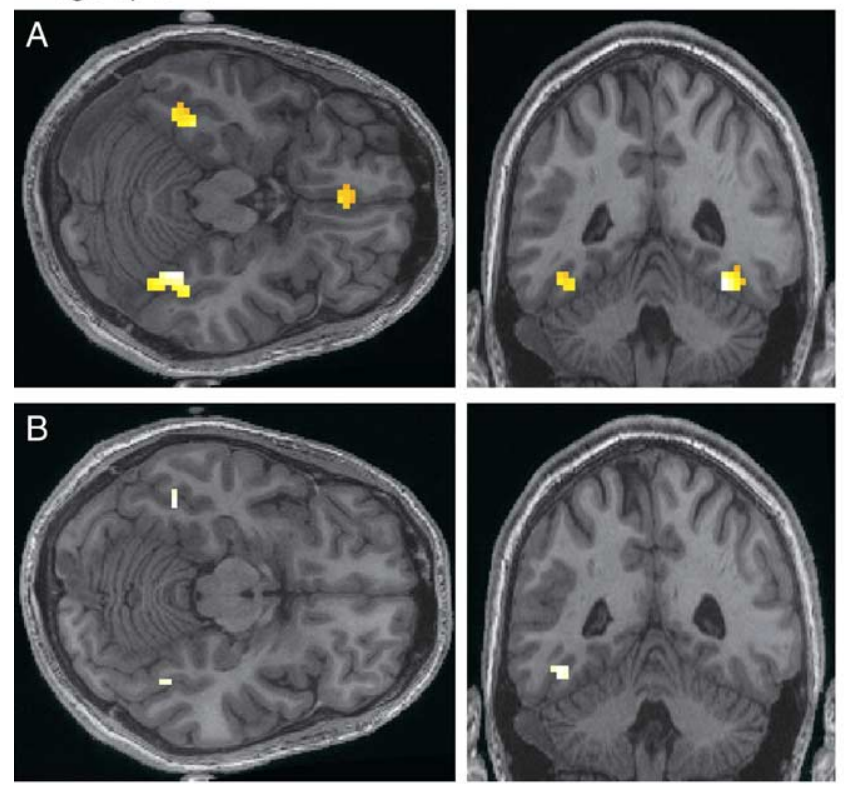

\section{Image-independent}
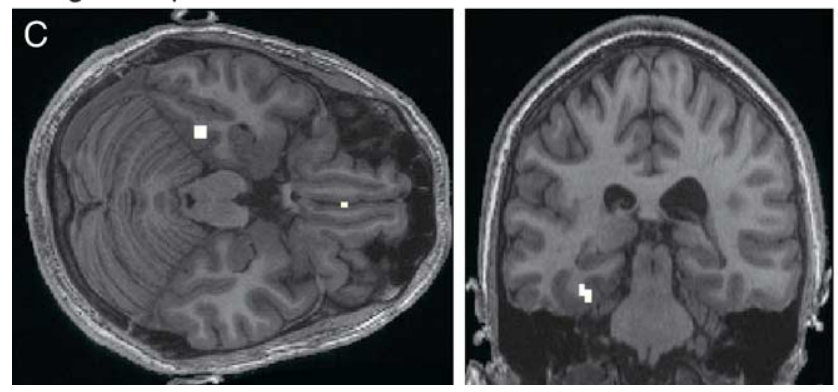
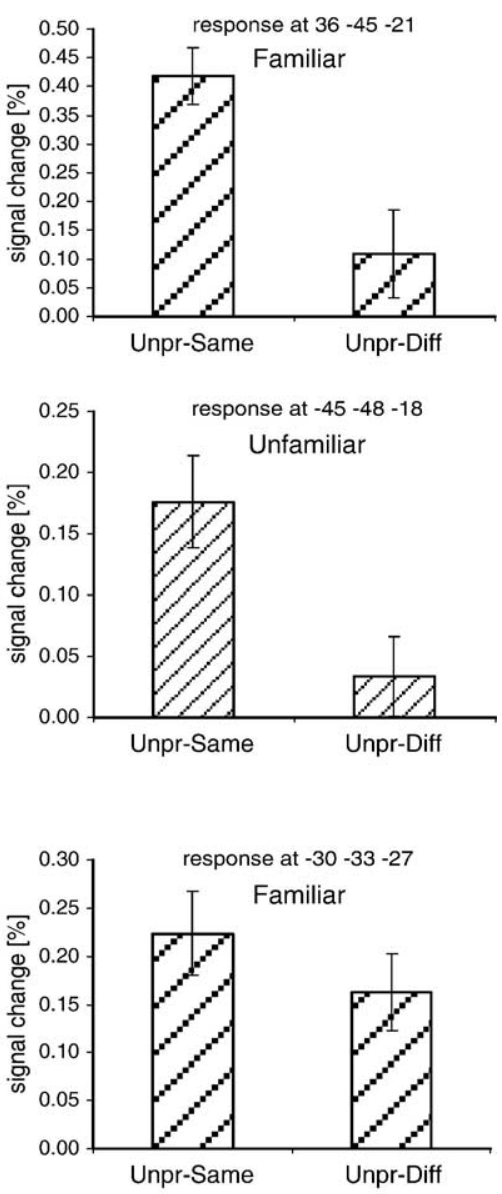

Fig. 4. Simple effect comparisons for image-dependent (unprimed $>$ same image, masked exclusively by unprimed $>$ different image at $P<0.001$ ) and imageindependent (unprimed $>$ different image, masked inclusively by unprimed $>$ same image) effects. T-contrasts for familiar (A,C) and unfamiliar (B) faces from the group analysis $(n=15)$ are overlaid on the structural scan of one participating subject. For visualisation purposes, effects in panel (A) were thresholded at $P<0.001$, uncorrected, and in panels (B) and (C) at $P<0.002$, uncorrected. All comparisons were masked inclusively by the main effect of priming and the faces localiser (at $P<0.001$, uncorrected). The plots give mean percentage of signal change and SEM for the most significant ventral visual foci corresponding to each comparison (same image and different image, subtracted from the unprimed baseline).

With regard to our first prediction of less image dependency of priming for familiar than unfamiliar faces, our reaction time data showed instead similar-sized priming effects for familiar and unfamiliar faces (i.e., no evidence of an interaction between familiarity and prime condition). This contrasts with the results of Roberts and Bruce (1989) who found priming across view changes only for familiar faces. Task factors (the present sex-decision task versus the detection of target faces used by Roberts and Bruce) or design factors (immediate repetition versus the one intervening item used by Roberts and Bruce) may contribute to this discrepancy. These behavioural measures of priming may nonetheless reflect contributions from facilitated processing in multiple different brain regions.

In the whole brain analysis, we did find an interaction between familiarity and priming in a medial anterior right fusiform region (medial and anterior to that found by Henson et al., 2000, 2002, 2003). This region showed greater priming-related reductions for familiar than unfamiliar faces, though this appeared most marked for the same image condition (Fig. 3). Most of bilateral middle fusiform cortex on the other hand showed evidence of priming- related reductions for both familiar and unfamiliar faces, as indicated by the main effect of priming (Table 2; Fig. 2). Priming-related reductions in fusiform cortex have not previously been found for unfamiliar faces (Henson et al., 2000, 2002, 2003), though one important difference between the present study and these previous ones is the present use of immediate rather than long-lag repetition (see, for example, Bentin and Moscovitch, 1988).

This pattern of priming effects across fusiform cortex was consistent with our second prediction of greater image independence in more anterior fusiform regions. In particular, the pattern observed in our ROI analysis was that image-independent repetition decreases for familiar faces were greater in anterior than middle fusiform cortex, whereas no such effects reached significance for the unfamiliar faces. This finding is compatible with an increasing abstraction of representations along the hierarchy of the ventral visual pathway and the role of familiarity in the achievement of this more invariant coding. Along a similar line, GrillSpector et al. (1999) showed that image independence increased along the ventral visual pathway (with respect to size and location 

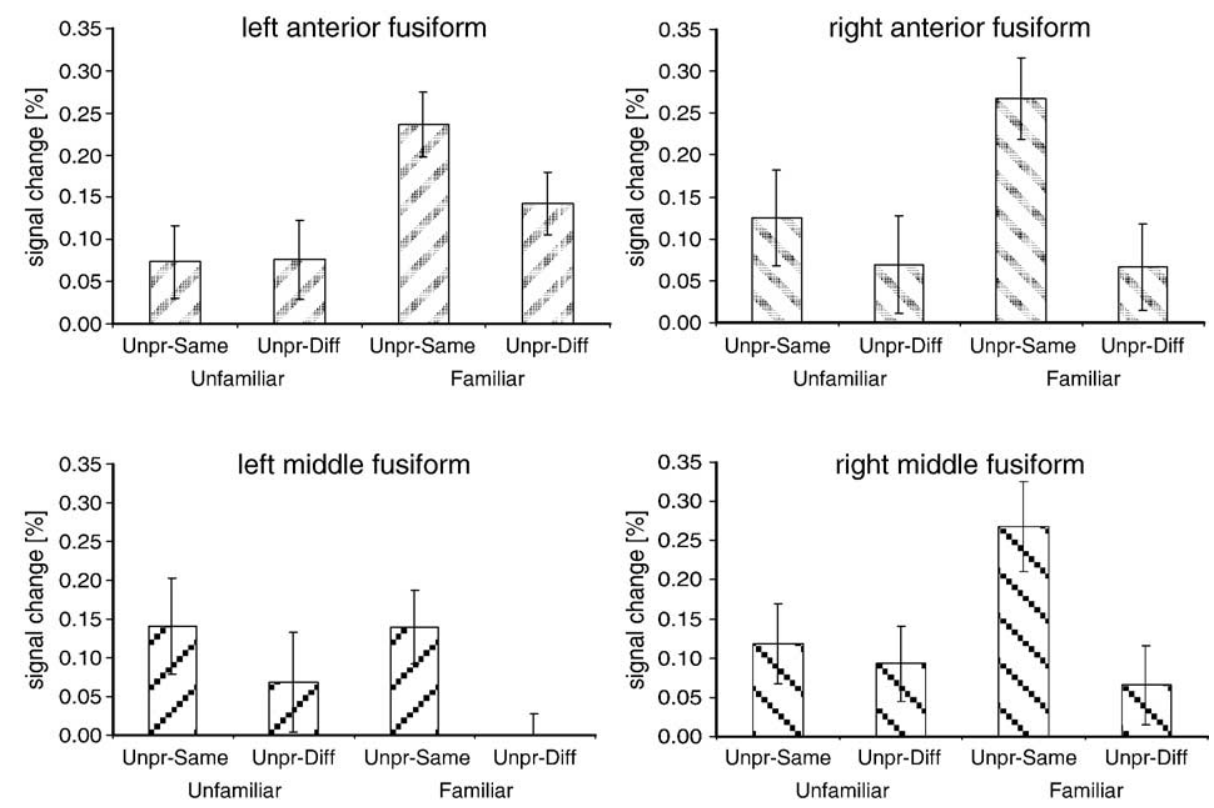

Fig. 5. Overview of regions of interest (ROI) data extracted from middle and anterior fusiform group maxima in the face localiser. Mean percentage of signal change and SEM is displayed for same and different image conditions subtracted from the unprimed baseline, for unfamiliar and familiar faces. A $2 \times 2 \times 2 \times$ 3 ANOVA with the factors of laterality, rostrality, familiarity, and prime condition revealed a significant 3-way interaction of rostrality $\times$ familiarity $\times$ priming. Follow-up analyses confirmed that, for familiar faces only, image-independent effects (unprimed - different image) were more pronounced in anterior than middle fusiform ROIs. The overall image-dependent effects (unprimed - same image), and the difference in priming between same and different images did not vary much across anterior and posterior regions. There was also a three-way interaction of laterality $\times$ rostrality $\times$ priming. While the overall image-dependent priming effect (unprimed - same image) was stronger on the right than left, the difference between same and different image priming was only greater for left than right in anterior fusiform.

of the face) when posterior and anterior parts of the LOC were compared. Generalisation across viewing angle was, however, not observed in their study (nor a more recent fMRI adaptation study by Andrews and Ewbank, 2004). Beyond differences in the experimental design, this could be related to the more pronounced differences in the rotational angle used in both studies or to their employment of only unfamiliar face pictures, as suggested by the present results.

With regard to our third prediction, the ROI analysis revealed a laterality difference in the anterior fusiform gyrus with respect to the degree of image dependence, where the relative size of the repetition decrease due to same as compared to a different image was enhanced on the right compared to the left (collapsing across familiar and unfamiliar faces). This finding is akin to the result obtained by Vuilleumier et al. (2002) for familiar objects (we note that, although this study reported a significant view-independent priming effect only on the left, a laterality difference in comparisons between regions was found significant only for the view-dependent effects, as in our data). More generally, it is consistent with the hemispheric lateralisation theory of priming exposed by Marsolek (1999). It also suggests that faces are processed similarly to objects in these regions and that any righthemisphere specialisation for faces (Landis et al., 1986; Rhodes, 1985) does not affect this type of modulation of priming-related neural responses by image/view.

\section{Further consideration of the effects of image changes}

We found both image-dependent (Same versus Unprimed conditions) and image-independent (Different versus Unprimed conditions) behavioural priming effects, where same image priming leads to an additional significant advantage of $45 \mathrm{~ms}$ (collapsing across familiarity). In the fMRI data also, imagedependent repetition effects seemed more pronounced than imageindependent effects. In the whole brain analyses, image-dependent repetition decreases were detected in bilateral fusiform cortex for familiar and unfamiliar faces, whereas image-independent repetition decreases reached significance only for familiar faces in a left fusiform region and in orbitofrontal cortex.

Although we found significant differences in the degree of image-dependence across subregions of the fusiform in our ROI analyses, the effect sizes (Fig. 5) suggest that most ROIs expressed a graded response pattern across the same image, different image, and different identity (unprimed) conditions rather than a clear segregation of subregions responding in an either fully imagedependent or image-independent fashion. This seems plausible given evidence from monkey single cell electrophysiology that neurons that respond specifically to a certain view of a face, and neurons that generalise across different views, coexist within the same macroscopic regions (see Rolls, 2000, for a review).

Generalisation across image changes could in principle reflect similarity of pictorial codes rather than extraction of a viewindependent representation. A mere overlap of pictorial information, however, is less likely to account for the finding of a differential effect for familiar versus unfamiliar faces (assuming that the images used for familiar and unfamiliar faces were comparable). Though there was no explicit control for the specific changes applied, our measure of image dissimilarity (see Results) does suggest that low-level image differences are unlikely to account for the more pronounced image-independent effects for familiar faces, since the tendency observed was in the opposite direction (the two photographs were more dissimilar for famous 
than unfamous faces). Nonetheless, our data do not fully resolve the extent to which the representations established from a single presentation (of an unfamiliar face) are abstract or pictorial. They are simply sufficient to produce some generalisation over image changes.

While our study showed the degree to which cortical regions express generalisation across different images as measured by fMRI, our data do not distinguish whether the neural mechanism underlying this generalisation reflects a single, abstract objectcentered representation or a pooling over several view-dependent representations. Both mechanisms have been proposed for objects (see e.g., Biederman and Kalocsai, 1997; Buelthoff et al., 1995), although abstract representations based on simple geometric primitives (e.g., Biederman, 1987) are generally agreed to be less able to account for the demands of face recognition than they are for basic-level object recognition.

Faces with which one is familiar have usually been seen from many different views, and thus the advantage of identifying them across images may result from the integration of these multiple "views" into a single representation. Behaviourally, it has been shown that the availability of different images can improve identification of unfamiliar faces, which is otherwise poor (Bruce et al., 2002). The idea of association of different views is further supported by evidence suggesting that temporal information (encountering the different views as they arise from natural movements of the head or face) plays an important role in this process: Wallis and Bulthoff (2001) used morphs of unfamiliar face stimuli that changed identity while the head rotated and observed that subjects were subsequently more likely to treat these views as if they were from the same person. In terms of neurophysiological substrate, it has been proposed (see Rolls, 2000) that prolonged firing of inferotemporal neurons after stimulus presentation functions as a short-term memory trace that is necessary to bind different retinal projections of an object into a common representation, which will consequently become viewpoint-invariant. It would be interesting to track this process of familiarisation of new objects or faces with functional imaging, which could then provide further insight into the nature of view-invariant object and face representations in ventral visual cortex. Such experiments would also be of interest because at the moment (using pictures of celebrities) our finding does not allow us to measure how much of the enhanced priming effects observed are due to purely visual familiarity rather than additional person knowledge. Studies using prefamiliarisation with new faces could help to disambiguate these two potential contributions.

\section{Other findings}

With regard to the main effects of faces versus scrambled faces and familiar versus unfamiliar faces, the present imaging results resemble those of Henson et al. (2003). In addition to lateral occipital and fusiform cortex, it is noteworthy that faces activated hippocampus, orbitofrontal cortex, and superior temporal sulcus bilaterally, relative to scrambled faces. These regions are consistent with evidence from single-cell recording in the nonhuman and human primate (Fried et al., 2002; Rolls, 1992; Scalaidhe et al., 1999). Bilateral temporal poles, orbitofrontal cortex, left fusiform and left hippocampus, and the retrosplenial/posterior cingulate cortex were further activated for familiar relative to unfamiliar faces. The importance of the temporal poles in person knowledge is well-documented from neuropsychology (Damasio et al., 1990).
Activation of the posterior cingulate cortex has been repeatedly observed for famous as compared to unfamous faces and sometimes voices (see e.g., Kosaka et al., 2003; Shah et al., 2001). The familiarity effect in the left hippocampus was less expected, though consistent with a recent fMRI study by Bernard et al. (2004). Note also that differences between familiar and unfamiliar faces may also exist in other regions, such as middle temporal gyrus (GornoTempini et al., 1998), that happened not to survive the threshold for our localiser contrast of the average of familiar and unfamiliar faces versus scrambled faces. Interestingly, a right hippocampal region showed an interaction between familiarity and priming, with a pattern of response reductions for familiar, but not unfamiliar, faces that resembled that in the medial anterior right fusiform region (Fig. 3). Finally, priming-related repetition decreases (image-dependent and image-independent) were also observed in orbitofrontal cortex. Priming effects in hippocampal and orbitofrontal cortex have not been observed previously (Henson et al., 2000, 2003), though again this may reflect the present use of immediate rather than long-lag repetition (Henson et al., 2004).

\section{Conclusion}

To summarise, the present study investigated priming-related repetition decreases for faces in ventral visual cortex according to their dependence on pictoral information and on face familiarity. The pattern observed in the ROI analysis, of stronger image specificity in the right than left anterior fusiform gyrus, confirms previous results obtained with non-face objects. Furthermore, an increasing degree of generalisation over image changes was found when progressing from mid- to anterior face-responsive regions in the fusiform, but only for familiar faces. Our findings thus support a critical role of experience in establishing more abstract cortical representations of face identities, which might provide a substrate for our superior abilities in identifying familiar as opposed to unfamiliar people.

\section{Acknowledgments}

This work was funded by Wellcome Trust Fellowship 060924 awarded to RH. RD is supported by the Wellcome Trust. We thank J. Kaufmann for his help in preparing the stimulus material used in this experiment.

\section{References}

Andrews, T.J., Ewbank, M.P., 2004. Distinct representations for facial identity and changeable aspects of faces in the human temporal lobe. NeuroImage 23, 905-913.

Ashburner, J., Friston, K.J., 1999. Nonlinear spatial normalization using basis functions. Hum. Brain. Mapp. 7, 254-266.

Bentin, S., Moscovitch, M., 1988. The time course of repetition effects for words and unfamiliar faces. J. Exp. Psychol. Gen. 117, $148-160$.

Bernard, F.A., Bullmore, E.T., Graham, K.S., Thompson, S.A., Hodges, J.R., Fletcher, P.C., 2004. The hippocampal region is involved in successful recognition of both remote and recent famous faces. NeuroImage 22, $1704-1714$.

Biederman, I., 1987. Recognition by components: a theory of human image understanding. Psychol. Rev. 94, 115-147. 
Biederman, I., Kalocsai, P., 1997. Neurocomputational bases of object and face recognition. Philos. Trans. R. Soc. Lond., B Biol. Sci. 352, $1203-1219$

Bruce, V., Henderson, Z., Greenwood, K., Hancock, P.J.B., Burton, A.M., Miller, P., 1999. Verification of face identities from images captured on video. J. Exp. Psychol., Appl. 5, 339-360.

Bruce, V., Ness, H., Hancock, P.J., Newman, C., Rarity, J., 2002. Four heads are better than one: combining face composites yields improvements in face likeness. J. Appl. Psychol. 87, 894-902.

Buelthoff, H.H., Edelman, S.Y., Tarr, M.J., 1995. How are three-dimensional objects represented in the brain? Cereb. Cortex 5, 247-260.

Damasio, A.R., Tranel, D., Damasio, H., 1990. Face agnosia and the neural substrates of memory. Annu. Rev. Neurosci. 13, 89-109.

Eger, E., Schyns, P., Kleinschmidt, A., 2004. Scale invariant adaptation in fusiform face responsive regions. NeuroImage 22, 232-242.

Ellis, A.W., Young, A.W., Flude, B.M., Hay, D.C., 1987. Repetition priming of face recognition. Q. J. Exp. Psychol. 39A, 193-210.

Fried, I., Cameron, K.A., Yashar, S., Fong, R., Morrow, J.W., 2002. Inhibitory and excitatory responses of single neurons in the human medial temporal lobe during recognition of faces and objects. Cereb. Cortex 12, 575-584.

Gauthier, I., Tarr, M.J., Anderson, A.W., Skudlarski, P., Gore, J.C., 1999. Activation of the middle fusiform "face area" increases with expertise in recognizing novel objects. Nat. Neurosci. 2, 568-573.

Gorno-Tempini, M.L., Price, C.J., Josephs, O., Vandenberghe, R., Cappa, S.F., Kapur, N., Frackowiak, R.S., Tempini, M.L., 1998. The neural systems sustaining face and proper-name processing. Brain 121, 2103-2118.

Grill-Spector, K., Kushnir, T., Edelman, S., Avidan, G., Itzchak, Y., Malach, R., 1999. Differential processing of objects under various viewing conditions in the human lateral occipital complex. Neuron 24, $187-203$.

Hancock, P.J.B., Bruce, V., Burton, A.M., 2000. Recognition of unfamiliar faces. Trends Cogn. Sci. 4, 330-337.

Henson, R.N., 2003. Neuroimaging studies of priming. Prog. Neurobiol. $70,53-81$.

Henson, R., Shallice, T., Dolan, R., 2000. Neuroimaging evidence for dissociable forms of repetition priming. Science 287, 1269-1272.

Henson, R.N., Shallice, T., Gorno-Tempini, M.L., Dolan, R.J., 2002. Face repetition effects in implicit and explicit memory tests as measured by fMRI. Cereb. Cortex 12, 178-186.

Henson, R.N.A., Goshen-Gottstein, Y., Ganel, T., Otten, L.J., Quayle, A., Rugg, M.D., 2003. Electrophysiological and haemodynamic correlates of face perception, recognition and priming. Cereb. Cortex $13,793-805$

Henson, R.N., Rylands, A., Ross, E., Vuilleumeir, P., Rugg, M.D., 2004. The effect of repetition lag on electrophysiological and haemodynamic correlates of visual object priming. NeuroImage 21, 1674-1689.

Hoffman, E.A., Haxby, J.V., 2000. Distinct representation of eye gaze and identity in the distributed human neural system for face processing. Nat. Neurosci. 3, 80-84

James, T.W., Humphrey, G.K., Gati, J.S., Menon, R.S., Goodale, M.A., 2002. Differential effects of viewpoint on object-driven activation in dorsal and ventral streams. Neuron 35, 793-801.

Kanwisher, N., McDermott, J., Chun, M.M., 1997. The fusiform face area: a module in human extrastriate visual cortex specialized for face perception. J. Neurosci. 17, 4302-4311.

Kosaka, H., Omori, M., Iidaka, T., Murata, T., Shimoyama, T., Okada, T., Sadato, N., Yonekura, Y., Wada, Y., 2003. Neural substrates participating in acquisition of facial familiarity: an fMRI study. NeuroImage 20, $1734-1742$

Koutstaal, W., Wagner, A.D., Rotte, M., Maril, A., Buckner, R.L., Schacter, D.L., 2001. Perceptual specificity in visual object priming: functional magnetic resonance imaging evidence for a laterality difference in fusiform cortex. Neuropsychologia 39, 184-199.
Landis, T., Cummings, J.L., Christen, L., Bogen, J.E., Imhoff, H.G., 1986. Are unilateral right posterior cerebral lesion sufficient to cause prosopagnosia? Clinical and radiological findings in six additional patients. Cortex 22, 243-252.

Malach, R., Reppas, J.B., Benson, R.R., Kwong, K.K., Jiang, H., Kennedy, W.A., Ledden, P.J., Brady, T.J., Rosen, B.R., Tootell, R.B., 1995. Object-related activity revealed by functional magnetic resonance imaging in human occipital cortex. Proc. Natl. Acad. Sci. U. S. A. 92, 8135-8139.

Marsolek, C.J., 1995. Abstract visual-form representations in the left cerebral hemisphere. J. Exp. Psychol. Hum. Percept. Perform 21, $375-386$

Marsolek, C.J., 1999. Dissociable neural subsystems underlie abstract and specific object recognition. Psychol. Sci. 10, 111-118.

Marsolek, C.J., Kosslyn, S.M., Squire, L.R., 1992. Form-specific visual priming in the right cerebral hemisphere. J. Exp. Psychol. Learn. Mem. Cognit. 18, 492-508.

Naccache, L., Dehaene, S., 2001. The priming method: imaging unconscious repetition priming reveals an abstract representation of number in the parietal lobes. Cereb. Cortex 11, 966-974

Puce, A., Allison, T., Gore, J.C., McCarthy, G., 1995. Face sensitive regions in human extrastriate cortex studied by functional MRI. J. Neurophysiol. 74, 1192-1199.

Rhodes, G., 1985. Lateralised processes in face recognition. Br. J. Psychol. $76,249-271$

Roberts, T., Bruce, V., 1989. Repetition priming of face recognition in a serial choice reaction-time task. Br. J. Psychol. 80, 201-211.

Rolls, E.T., 1992. Neurophysiological mechanisms underlying face processing within and beyond the temporal cortical visual areas. Phil. Trans. R. Soc. Lond., B 355, 11-20.

Rolls, E.T., 2000. Functions of the primate temporal lobe cortical visual areas in invariant object and face recognition. Neuron 27, $205-218$

Scalaidhe, S.P., Wilson, F.A., Goldman-Rakic, P.S., 1999. Face-selective neurons during passive viewing and working memory performance of rhesus monkeys: evidence for intrinsic specialization of neuronal coding. Cereb. Cortex 9, 459-475.

Schweinberger, S.R., Pfuetze, E.M., Sommer, W., 1995. Repetition priming and associative priming of face recognition: evidence from event-related potentials. J. Exp. Psychol. Learn. Mem. Cognit. 21, 722-736.

Schweinberger, S.R., Pickering, E.C., Jentzsch, I., Burton, A.M., Kaufman, J.M., 2002. Event-related potential evidence for a response of inferior temporal cortex to familiar face repetitions. Cogn. Brain Res. 14, 398-409.

Sergent, J., Ohta, S., MacDonald, B., 1992. Functional neuroanatomy of face and object recognition. Brain 115, 15-36.

Shah, N.J., Marshall, J.C., Zafiris, O., Schwab, A., Zilles, K., Markowitsch, H.J., Fink, G.R., 2001. The neural correlates of person familiarity. A functional magnetic resonance imaging study with clinical implications. Brain 124, 804-815.

Simons, J.S., Koutstaal, W., Prince, S., Wagner, A.D., Schacter, D.L., 2003. Neural mechanisms of visual object priming: evidence for perceptual and semantic distinctions in fusiform cortex. NeuroImage $19,612-626$.

Srinivas, K., 1993. Perceptual specificity in nonverbal priming. J. Exp. Psychol. Learn. Mem. Cognit. 19, 582-602.

Tarr, M.J., Cheng, Y.D., 2003. Learning to see faces and objects. Trends Cogn. Sci. 7, 23-30.

Vuilleumier, P., Henson, R.N., Driver, J., Dolan, R.J., 2002. Multiple levels of visual object constancy revealed by event-related fMRI of repetition priming. Nat. Neurosci. 5, 491-499.

Wallis, G., Bulthoff, H.H., 2001. Effects of temporal association on recognition memory. Proc. Natl. Acad. Sci. U. S. A. 98, 4800-4804.

Wells, G.L., 1993. What do we know about eyewitness identification? Am. Psychol. 48, 553-571. 